\title{
Effective Pressure for Two Phase Water-Sediment Models using SPH Method
}

\author{
M. Kheirkhahan ${ }^{1}$, P. Omidvar ${ }^{2 \dagger}$ and K. H. Hosseini ${ }^{1}$ \\ ${ }^{1}$ Faculty of Civil Engineering, Semnan University, Semnan, Iran \\ ${ }^{2}$ Faculty of Engineering, Yasouj University, Yasouj, Iran \\ †Corresponding Author Email: omidvar@yu.ac.ir
}

(Received October 15, 2019; accepted December 15, 2019)

\begin{abstract}
Sediment transport in the aquatic environment is one of the complex two-phase problems in flow mechanics and sediment hydraulics. In this study, the interaction between water and sediment is explored using the SPH method and developed SPHysics2D in which the pressure values are calculated using the state equation. In this study, the non-Newtonian rheological model $\mu(I)$ is used for modeling the sediment phase where it is developed based on the properties of granular particles. Also, the effective pressure is used for the study of sediment behavior. The method used in this research is compared with the methods used by other researchers. The Owen equation is utilized to determine the effect of viscosity within the two-phase area. The developed method is evaluated by the dam break on a dynamic bed and then, the experimental model of submerged sediment column collapse is investigated in the aquatic environment. The results of the modeling demonstrate the capabilities of the developed code for the use in the flow and sediment hydraulics.
\end{abstract}

Keywords: Rheological model; Owen model; Effective pressure; Dam break with movable bed; Submerged column collapse.

\section{NOMENCLATURE}

$C_{I} \quad$ constant

$C_{S} \quad$ Smagorisky constant

$c_{0} \quad$ sound speed

D particle diameter

$F \quad$ force

$g$ gravity acceleration

$h$ height

I inertia

$K \quad$ coefficient

$l \quad$ length

$m$ particle mass

$P \quad$ pressure

\section{INTRODUCTION}

Rapid flow hydraulics on a fluvial bed leads to huge changes in the river morphology. River morphology, in turn, influences the hydraulic parameters of current. Thus, modeling the rapid flow with sediment transport is a complicated issue in the fluid mechanics. Dam break is known as a rapid flow phenomenon accompanied by the huge deformation in the water surface profile which induces great deformations on the bed. Close interactions exist

$\begin{array}{ll}P_{e f f} & \text { effective pressure } \\ P_{p w} & \text { pore water pressure } \\ R & \text { balance factor } \\ t & \text { time } \\ u & \text { velocity } \\ W & \text { interpolation function } \\ & \\ \dot{\gamma} & \text { strain tensor } \\ \mu & \text { friction } \\ v & \text { viscosity } \\ \rho & \text { density } \\ \tau & \text { shear stress }\end{array}$

between hydrodynamics, sediment transport and bed morphology, Moreover, the nonlinear sediment behavior caused by the stress induced by current makes the problem more complicated.

Submerged soil column collapse is an example of water-soil interaction problems, which has an application in the debris flows (Iverson, 1997), landslides (Legros, 2002), sub-marine avalanches (Hampton et al., 1996), etc. Since it may have harmful impacts on the safety of the underwater structures or the geo-morphological changes of 
waterways, the submerged granular column collapse has received particular interest.

Due to the complex interaction between water and solid particles, the process of submerged soil column collapse has not yet been well understood in comparison with the dry soil column collapse. Rondon et al. (2011), Meruane et al. (2010, 2012), Savage et al. (2014) recently investigated this issue, showing the challenges and developments.

The mathematical models capable of accurately simulating both the bed and water surface profiles are limited. Computational Fluid Dynamics (CFD) based on the Eulerian approaches has made great progresses on modeling two-phase non-Newtonian flows. However, the Eulerian and mesh-based numerical methods may have difficulties with the moving boundaries (Shadloo et al. 2016).

The Lagrangian method is another approach to model the current by solving the Navier-Stokes and continuity equations. Smoothed Particle Hydrodynamics (SPH) is a mesh-free and fully Lagrangian method where the fluid is divided into a set of particles interacting with each other (Gingold and Monaghan, 1977; Lucy, 1977). The Lagrangian methods are widely utilized in modeling the freesurface flows (Omidvar et al. 2015), wave-body interaction (Omidvar et al. 2017), multiphase flows (Hosseinkhani and Omidvar, 2018), and nonNewtonian fluid flows (Shao and Lo, 2003; Shakibaeinia and Jin, 2011; Khanpour et al. 2016; Omidvar et al. 2018).

The movement of granular materials and noncohesive sediments is accomplished by exceeding the shear stress imposed on the particle from a threshold value called the yield stress. If the shear stress is less than the yield stress, the particle remains motionless and the movement is started beyond the yield stress. Therefore, the particles can be modeled as a non-Newtonian fluid that technically has certain viscoplastic behavior. Some known viscoplastic models with a particular yield stress for the motion threshold are: HerschelBulkley, Cross, Bingham, etc. (Khanpour et al. 2016; Omidvar and Nikeghbali, 2017; Fourtakas and Rogers, 2016). Szewc (2017) implemented the Cross rheological model in SPH code developed for numerical models. He obtained a good agreement by comparing with experimental results. Moving Particle Semi-implicit (MPS) is another Lagrangian model which was applied to model the dam-break phenomenon on a moving bed by Fu and Jin (2016). The Herschel-Bulkley (HB) rheological model was used in the sediment phase. A two-phase watersediment model was developed using the Weakly Compressible SPH (WCSPH) by Fourtakas and Rogers (2016). Khanpour et al. (2016) used the WCSPH to model the scouring and flushing phenomena. They proposed the Bingham model to simulate the behavior of sediment phase. The influence of violent water flow on a loose boundary was also investigated by Omidvar and Nikeghbali (2017) through the SPH model. In this research, the Bingham model was considered as the rheological model. They could determine the water-solid interface using the concentration of solid in each section (Nikeghbali and Omidvar, 2018). Kheirkhahan and Hosseini (2018) applied the HBP (Herschel-Bulkley-Papanastasiou) and $\mu(I)$ rheological models for simulating the motion of granular particles through the SPH model. Also, Hosseini et al. (2019) found reasonable performance for $\mu(I)$ rheological model by simulating the dam break and landslide phenomena on a moving bed.

An open-source SPHysics2D code is available for modeling Newtonian fluids (Go'mez-Gesteria et al. 2012). In this research and after Omidvar et al. (2018) and Hosseini et al. (2019)", the $\mu(I)$ nonNewtonian model is used to study the motion of particles where the effective pressure of sediment phase is calculated in each time step by subtracting the water pressure from total sediment pressure. For simulating this phenomenon, the gate supporting the particles in the reservoir is instantaneously removed to study the motion of particles. First, the granular dam break phenomenon is studied by numerical approach implemented in SPHysics2D code The pressure resulting from this method is compared with the effective pressure suggested by Fourtakas and Rogers (2016), and the results show better performance. After ensuring the code function by comparing the results of experimental model, the code is developed for modeling the dam break on an moving bed. In the two-phase models, some complicity appears due to the differences in viscosity, density and other parameters in two phases. Grenier et al. (2009) proposed the relations to overcome the singularities concerned to the difference in density at the interface of two phases. The effect of differences in viscosity of two phases at the interfaces is investigated by Owen. All modifications by Grenier et al. (2009) and Owen are utilized at the interface.

\section{Governing EQUATIONS}

Continuity and momentum equations for fluid flow expressed in Lagrangian form are:

$\frac{D \rho}{D t}+\rho(\nabla \cdot \vec{u})=0$

$\rho \frac{D \vec{u}}{D t}=-\nabla P+\nabla \tau+\vec{F}$

in which, $\rho$ density, $\vec{u}$ is velocity vector, $P$ is pressure, $\tau$ stress tensor and $\vec{F}$ other body forces influencing the movement of flow and $t$ is time.

\section{SPH METHOD}

In this method, the particles of fluid behave as continuum media, so the governing equations could be used for all particles in modeling the movement. SPH method is based on the movement of each particle influenced by the forces subjected by the other particles. This method profits the interpolation of integral function $f$ on the domain of $\Omega, f$ function is discrete, using convolution function (Gingold and Monaghan, 1977; Nikeghbali and Omidvar, 2018). 
$f(x) \approx<f(x)>$

$=\int_{\Omega} f\left(x^{\prime}\right) W\left(x-x^{\prime}, h\right) d x^{\prime}$

Equation (3) converted to Eq. (4) by introducing discrete particle approximation, called interpolation approximation.

$$
\begin{array}{r}
f\left(x_{i}\right)=\sum_{j} \frac{m_{j}}{\rho_{j}} f\left(x_{j}\right) W\left(x_{i}-x_{j}, h\right) \\
=\sum_{j} \frac{m_{j}}{\rho_{j}} f\left(x_{j}\right) W_{i j}
\end{array}
$$

in which $W_{i j}$ is interpolation function, $h$ smoothed height, $m$ and $\rho$ are mass and density of particle, respectively. Figure 1 shows the neighbors of particle $i$ within the interpolation function.

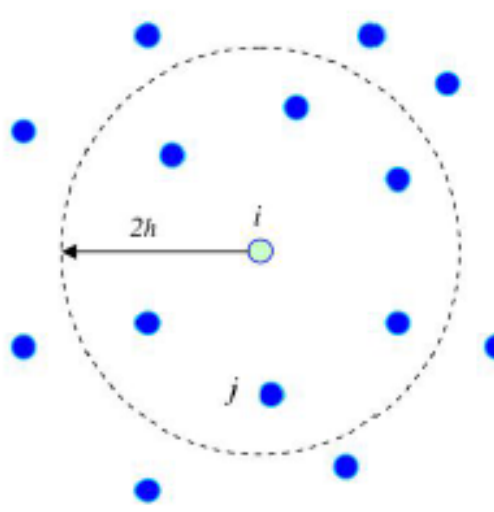

Fig. 1. Neighbors of particle $i$ within the interpolation function.

Therefore, main forms of governing equations in SPH method are showed in Eq. (5) and Eq. (6).

$$
\begin{aligned}
& \frac{D \rho_{i}}{D t}=\sum_{j} m_{j} v_{i j} \nabla_{i} W_{i j} \\
& \frac{D \vec{u}_{i}}{D t}=\frac{F}{\rho_{i}}-\sum_{j} m_{j}\left(\frac{P_{i}}{\rho_{i}{ }^{2}}+\frac{P_{j}}{\rho_{j}{ }^{2}}\right) \nabla_{i} W_{i j} \\
& \quad+\sum_{j} m_{j}\left(\frac{4 v r_{i j} \cdot u_{i j}}{\left(\rho_{i}+\rho_{j}\right)\left(\left|r_{i j}\right|^{2}+\iota^{2}\right)}\right) \nabla_{i} W_{i j} \\
& \quad+\sum_{j} m_{j}\left(\frac{\bar{\tau}_{i}}{\rho_{i}{ }^{2}}+\frac{\bar{\tau}_{j}}{\rho_{j}{ }^{2}}\right) \nabla_{i} W_{i j}
\end{aligned}
$$

where $P$ is the particle pressure, $v_{i j}$ is velocity difference of $i$ and $j$ particles, $v$ is fluid kinematic viscosity, $r_{i j}=\left|x_{i}-x_{j}\right|$, and $\iota$ is announced as a very small value for preventing relation infinity.

The first term at right side of Eq. (6) is body forces, in second term pressure gradient is defined, the third term defines viscosity proposed by Morris et al. (1997) and the last term is the turbulence stress which was applied by the Eq. (7) (Lo and Shao 2002; Nikeghbali and Omidvar, 2018; Gómez-Gesteria et al. 2012).

$$
\begin{aligned}
& \frac{\tau_{\alpha \beta}}{\rho}=v_{t}\left(2 \dot{\gamma}_{\alpha \beta}-\frac{2}{3} k \delta_{\alpha \beta}\right) \\
& -\frac{2}{3} C_{I} \Delta^{2} \delta_{\alpha \beta}\left|\dot{\gamma}_{\alpha \beta}\right|^{2}
\end{aligned}
$$

where, $\tau_{\alpha \beta}$ is the stress tensor of SPS (Sub-Particle Scale), $v_{t}$ turbulence viscosity defined in Smagorinsky's model as $v_{t}=\left[\min \left(C_{s} \Delta l\right)\right]^{2}\left|\dot{\gamma}_{\alpha \beta}\right|$ in which the Smagorisky constant is $C_{s}=0.12 \cdot \dot{\gamma}_{\alpha \beta}$ is shear rate tensor and $\left|\dot{\gamma}_{\alpha \beta}\right|$ is second invariant of shear rate tensor. $k$ is the turbulence viscosity of SPS and $C_{I}=0.0066$ (Nikeghbali and Omidvar, 2018).

The shear rate tensor is defined by Eq. (8) and its second invariant is defined by Eq. (9) (Nikeghbali and Omidvar, 2018)

$\dot{\gamma}_{\alpha \beta}=\nabla u+\nabla u^{T}$

and

$|\dot{\gamma}|=\sqrt{\frac{1}{2} \dot{\gamma}_{\alpha \beta} \dot{\gamma}_{\alpha \beta}}$

Grenier et al. (2009) proposed an artificial pressure for the particles interface with different densities to avoid instabilities where the second term in momentum equation is replaced by $\sum_{j} m_{j}\left(\frac{P_{i}}{\rho_{i}{ }^{2}}+\right.$ $\left.\frac{P_{j}}{\rho_{j}^{2}}+R_{i j}\right) \nabla_{i} W_{i j}$ for the interfacial particles with different densities. This term is shown by Eq. (10).

$R_{i j}=K\left(\frac{\rho_{\mathrm{d}}-\rho_{\mathrm{l}}}{\rho_{\mathrm{d}}+\rho_{\mathrm{l}}}\right)\left|\frac{P_{i}+P_{j}}{\rho_{i} \rho_{j}}\right|$

where $\rho_{\mathrm{d}}$ and $\rho_{\mathrm{l}}$ are equal to reference densities of fluids. $K$ is a coefficient which is considered between 0.01 and 0.1 .

Here, a third-order interpolation function (Hosseini et al, 2019) and the repulsive boundary condition (Monaghan and Kos 1999; Rogers et al. 2009) is used for the wall particles. Moreover, the Predictorcorrector scheme is used as a temporal scheme (Monaghan, 2005).

\section{RHEOlOGicAl Model}

Here, we used the $\mu(I)$ rheological model to simulate the motion of particles, which was first introduced by GDR MiDi (2004) and Da Cruz et al. (2005).

The relation between the shear and the normal stress in this model is defined by Eq. (11) (Xu and Jin, 2016).

$\tau=\mu p$

where $\mu$ is the coefficient of friction, $\tau$ is the shear stress and $p$ is the normal stress. Here, $\mu$ is dependent on the inertia according to Da Cruz et al. (2005):

$I=\frac{|\dot{\gamma}| D}{\sqrt{p / \rho_{s}}}$

where $|\dot{\gamma}|$ is the shear rate, $D$ is the particle diameter, $p$ is the pressure and $\rho_{s}$ is the density of particle. Moreover, Eq. (13) presents the value of friction coefficient $\mu$ (Jop et al. 2005). 


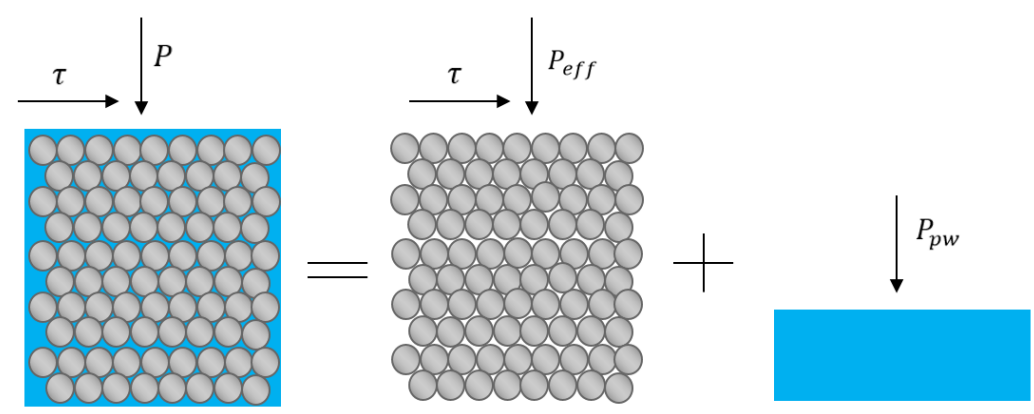

Fig. 2. A view of normal and shear stresses supported by different phases in a saturated soil.

$\mu=\mu_{s}+\frac{\mu_{2}-\mu_{s}}{1+I_{o} / I}$

$\mu_{s}, \mu_{2}$ and $I_{o}$ are the parameters related to each material and is determined in the lab ( $\mathrm{Xu}$ and Jin, 2016; Kheirkhahan and Hosseini, 2018).

Applying Eq. (11) in two and three dimensions results in Eq. (14) which relates shear stress to pressure.

$\tau_{\alpha \beta}=\frac{\mu(I) p}{|\dot{\gamma}|} \dot{\gamma}_{\alpha \beta}=\eta \dot{\gamma}_{\alpha \beta} \quad, \quad \eta=\frac{\mu(I) p}{|\dot{\gamma}|}$

which is practically found according to the experimental data and numerical models. The proposed regularization method of Papanastasiou (Papanastasiou, 1987) transposed to the $\mu(I)$ rheology (Chauchat and Médale, 2014).

$\eta_{r}$

$=\mu_{s} p \frac{1-e^{-|\dot{\gamma}| / \alpha_{r}}}{|\dot{\gamma}|}+\frac{\left(\mu_{2}-\mu_{s}\right) p}{I_{o} \sqrt{\phi p}+|\dot{\gamma}|+\alpha_{r}}$

where $0.6 \leq \phi \leq 0.65, \alpha_{r}$ is a small parameter to avoid zero value in the denominator and $p$ is the confining pressure (stress between granular particles). In the saturated soils, the effective pressure $\left(P_{\text {eff }}\right)$ appears in the $\mu(I)$ relation. In this research, $\phi$ and $\alpha_{r}$ are 0.62 and 0.000001, respectively (Chauchat and Médale, 2014; Xu and Jin, 2016).

The apparent viscosity $(\eta)$ for each particle depends on the position and the pressure term, as discussed above, and hence, the viscosity is relevant to each particle. Thus, the mean harmonic viscosity expressed by Eq. (16) gives more appropriate value for the viscosity (Hosseini et al. 2019):

$\eta_{i j}=\eta_{j i} \cong \frac{2 \eta_{i} \eta_{j}}{\eta_{i}+\eta_{j}}$

where subscript $i$ refers to the main particle and $j$ refers to the particle situated within the kernel (interpolation function) radius. For the particles belonging to the interface, the calculation of some parameters such as viscosity of mixture becomes difficult. The Owen equation allows to calculate the mixture viscosity considering the solid concentration described as below (Hosseini et al. 2019):

$$
\begin{aligned}
v_{\text {mix }} & =\frac{v_{\text {fluid }}}{1+C \frac{\rho_{s}}{\rho_{f}}} \\
C & =\frac{\sum_{j \neq i} \delta_{s f} W_{i j}}{\sum_{j \neq i} \delta_{s f} W_{i j}+\sum_{j \neq i}\left(1-\delta_{s f}\right) W_{i j}}
\end{aligned}
$$

where $\rho_{s}, \rho_{f}$ and $C$ are the water density, sediment density and concentration of solid, respectively (Hosseini et al. 2019). Eq. (18) is used to determine the viscosity in two-phase mixture, where $\delta_{s f}$ is replaced by zero for fluid and one for solid.

\section{Pressure Calculations}

The application of the WCSPH method is an approach to determine the pressure using the state equation defined as follows (Gingold and Monaghan, 1977; Monaghan, 1994; Khanpour et al. 2016).

$P_{i}=B\left(\left(\frac{\rho_{i}}{\rho_{o}}\right)^{\gamma}-1\right)+$ backpressure

In Eq. (20), $\gamma$ is constant and equal to $7, \rho_{o}$ is the reference density, $\rho_{i}$ is the particle density, and $B=$ $c_{o}{ }^{2} \rho_{o} / \gamma$.

For improving the calculation of pressure along the solid phases, Colagrossi and Landrini (2003) proposed to add backpressure term to the state equation. By introducing backpressure term, the pressure of the water column above the solid particle will be added to the pressure calculated from the state equation. In this paper, the backpressure term is considered.

\section{Effective Pressure}

Based on the Terzaghi's theory, when a saturated soil is subjected to the normal and shear stresses (Fig. 2), the stresses corresponding to each phase are as follows:

Water does not support any shear stress, but it supports part of the normal stress called pore pressure. Effective pressure, total pressure subtracted by pore pressure, refers to part of the normal stress supported by the solid structure of the soil. Based on the Mohr-Coulomb relation, the effective pressure is converted to the shear stress 
available in the soil, which resists to the shear stress. It should be noted that the sediments start moving if the total shear stress is more than the threshold stress.

As shown in Fig. 2, the pressure supported by the solid structure is calculated by subtracting the pore pressure from the total pressure. During the calculating process, the pore pressure is subtracted from the total pressure to obtain the effective pressure for solid particles, which can be introduced into the rheological equation (Eq. (15). In this paper, two methods are employed to determine the pore and effective pressures: the method developed by Fourtakas and Rogers (2016) and the method developed in this research. Fourtakas and Rogers (2016) proposed the following equation for the effective and pore pressures:

$P_{e f f}=P_{t}-P_{p w}$

where:

$P_{p w}=\frac{c_{o}^{2} \rho_{o w}}{\gamma}\left(\left(\frac{\rho_{i t}}{\rho_{o t}}\right)^{\gamma}-1\right)$

and $P_{e f f}$ is the effective pressure, $P_{t}$ is the total pressure from the state equation (Eq. (20)) for the solid phase and $P_{p w}$ is the pore pressure. $\rho_{i t}$ and $\rho_{o t}$ are the density of solid particle and reference density of the saturated soil, respectively. Other parameters were previously described.

The method developed in this research is founded on the concept of stresses induced on a saturated soil body. Based on the schematic view of the column of water and sediment shown in Fig. 3, the pressure imposed on the sediment particles situated at the distance $h_{2}$ under the water-sediment interface and water depth $h_{1}$ is defined by Eq. (22):

$P_{t}=P_{w}+P_{s a t}=\gamma_{w} h_{1}+\gamma_{s a t} h_{2}$

where $\gamma_{s a t}$ and $\gamma_{w}$ are the specific gravity of sediment and water, respectively. $P_{w}$ is the water pressure on the interface. The effective density of sediments is calculated by Eq. (23).

$\gamma_{e f f}=\gamma_{s a t}-\gamma_{w}$

Combining Eq. (22) and Eq. (23) leads to Eq. (24):

$P_{t}=P_{w}+P_{\text {sat }}$

$=\gamma_{w} h_{1}+\left(\gamma_{e f f}+\gamma_{w}\right) h_{2}$

$=\underbrace{\gamma_{w}\left(h_{1}+h_{2}\right)}_{P_{p w}}+\underbrace{\gamma_{\text {eff }} h_{2}}_{P_{\text {eff }}}$

where the effective pressure is obtained from Eq (25).

$P_{e f f}=P_{t}-P_{p w}$

In the above equation, $P_{t}$ and $P_{p w}$ are the pressure obtained from the numerical analysis and the pore water pressure, respectively. The shear stress is supported only by the solid portion of watersediment mixture, which is related to the effective pressure exerted on the particle. Thus, the effective pressure can be introduced into the rheological models.

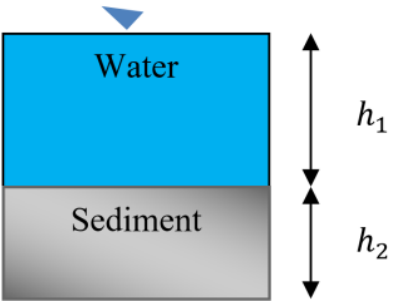

Fig. 3. A schematic view of water-sediment system.

\section{RESUlts And Discussion}

In this paper, the granular dam break experimental data has been utilized to investigate the application of the rheological model. The dam break problem on a movable bed is simulated to simulate where the performance of the modified model with a proposed effective pressure is shown and compared with other data.

\subsection{Modeling the Movement of Grains}

In this paper, the verification test for the numerical method based on the viscoplastic model is done using the experimental data published by Lajeunesse et al. (2005), where the granular dam break phenomenon is studied in laboratory for the glass balls with $D=1.15 \mathrm{~mm}, \rho_{o}=2500 \mathrm{~kg} / \mathrm{m}^{3}$ and $\theta=22^{\circ}$. The granular dam break is investigated by considering the following parameters: $a=h_{o} / l_{o}$ and $t^{*}=\sqrt{h_{o} / g}$, where the parameters are related to the initial height $\left(h_{o}\right)$ and initial length $\left(l_{o}\right)$ of the particle mass in the reservoir. The initial height to initial length ratio $(a)$ is an effective parameter in the motion of particles. For the same $a$ but different $h_{o}$ and $l_{o}$, the results for the motion pattern of particles are identical. For the verification test, the experimental data for $a=0.6$ and $l_{o}=0.053 \mathrm{~m}$ are compared with the numerical results. In the numerical model, the distance between the particles in $\mathrm{x}$ and $\mathrm{z}$ directions is defined as $2 \mathrm{~mm}$, which resulted to 3816 particles. The time taken for the simulation was 4412 seconds in a computer with Windows 10/CPU Intel core i7 6800K/RAM 16GB.

A schematic view of the rectangular reservoir is presented in Fig. 4. The materials are lean on a gate in the right side of the figure. The granular dam break is ensured by instantaneously moving the gate upward. The values corresponding to $\mu(I)$ model are: $I_{o}=0.279, \mu_{s}=\tan 20.90^{\circ}$ and $\mu_{2}=\tan 32.76^{\circ}$ (Lajeunesse et al. 2005; Xu and Jin, 2016). According to Kheirkhahan and Hosseini (2018), the velocity of gate opening is considered $0.8 \mathrm{~m} / \mathrm{s}$. In Fig. 5, the free surface of the wave in both experimental and numerical models produced from the granular dam break is compared for different times. The numerical results are consistent with the experimental data.

In Fig. 6, the position, velocity and pressure of particles are presented for different times. As shown in the figure, the motion of particles is started from the lowest point in the vicinity of the gate. 


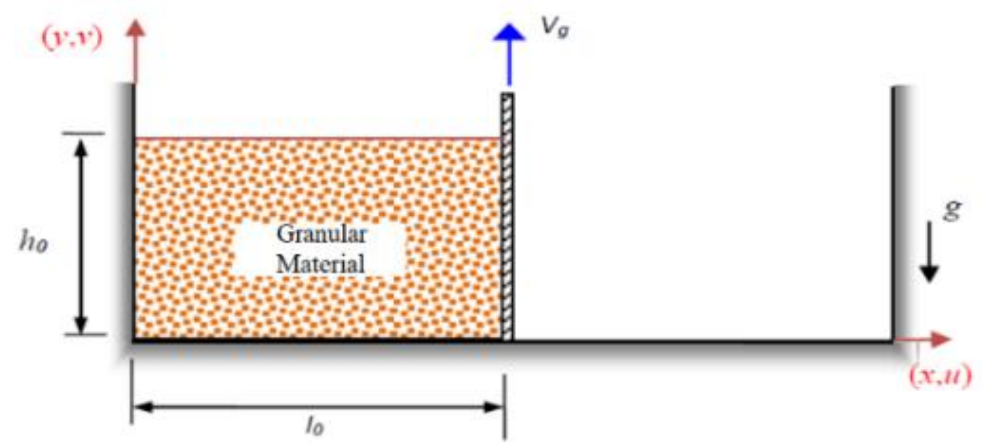

Fig. 4. A sketch view of reservoir and initial positions of granular particles.

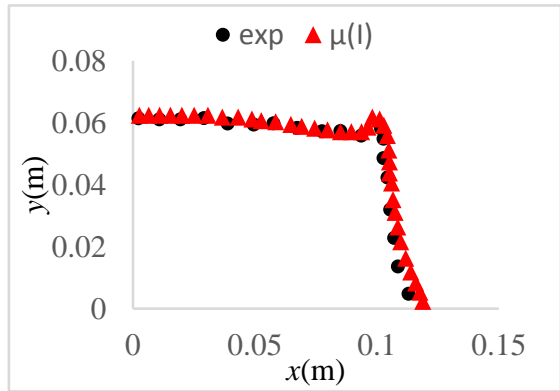

$t / t^{*}=1$

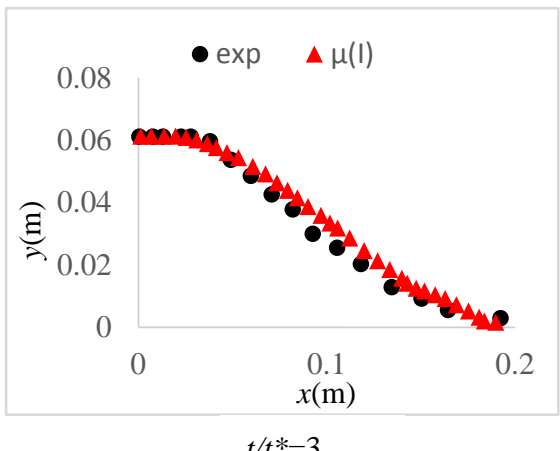

$t / t^{*}=3$

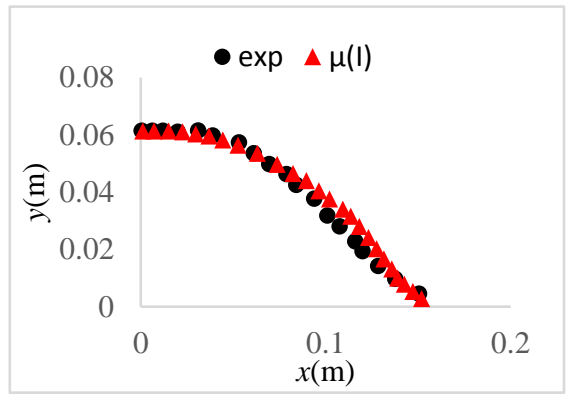

$t / t^{*}=2$

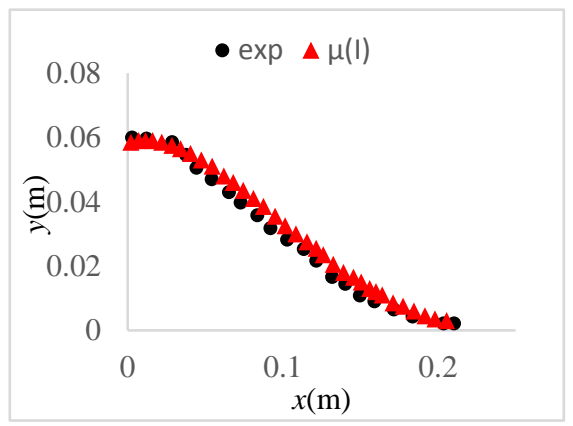

$t / t^{*}=4$

Fig. 5. Comparison the free surface of granular body in both experimental and numerical model.

Afterwards, the motion is developed by sliding the upper particles on the lower particles. In each time, the upper particles have the maximum velocity, and some particles situated between the bed and the left vertical wall remain motionless. The particles support the maximum pressure. It is concluded that the numerical model can simulate the motion of particles. As shown in Fig. 5, the particles subjected to the maximum pressure have the maximum shear strength. The particles are enclosed between the bed, the left wall and the upper mass. This behavior is relevant to the motion of the granular mass. The movement of granular body is started from the lower part of mass where the particles have the maximum velocities. As seen in the figure there is a gap between the leading edge particle and the boundary. The reason might be due to lack of particles in the kernel support and the repulsive force exerted by the boundary particles.

Figure 7 shows the values of the apparent viscosity.
As seen in the figure, the viscosity values vary in soil mass due to non-Newtonian behavior of particles, and the particles move in the places where these values are lower. In the latest time, the viscosity of the entire mass reaches a value that resists the forces penetrating the particle, and the particles stop moving.

\subsection{Water-Sediment Two-Phase Modeling of Dam Break on a Loose Boundary Grains}

By ensuring the performance of the numerical model in modeling the movement of granular materials, its performance for the two-phase model is investigated. Here, we use the experimental data of Spinewine (2005) for the validation. The rheological parameters of sediments are: $\rho_{s}=2683 \mathrm{~kg} / \mathrm{m}^{3}, \quad D=$ $1.82 \mathrm{~mm}, I_{o}=0.279, \mu_{s}=\tan 30.5^{\circ}$ and $\mu_{2}=$ $\tan 51.3^{\circ}$ (Spinewine, 2005; Xu and Jin, 2016) and the fluid phase consists of water with specific density $1000 \mathrm{~kg} / \mathrm{m}^{3}$ and the kinematic viscosity equals to 

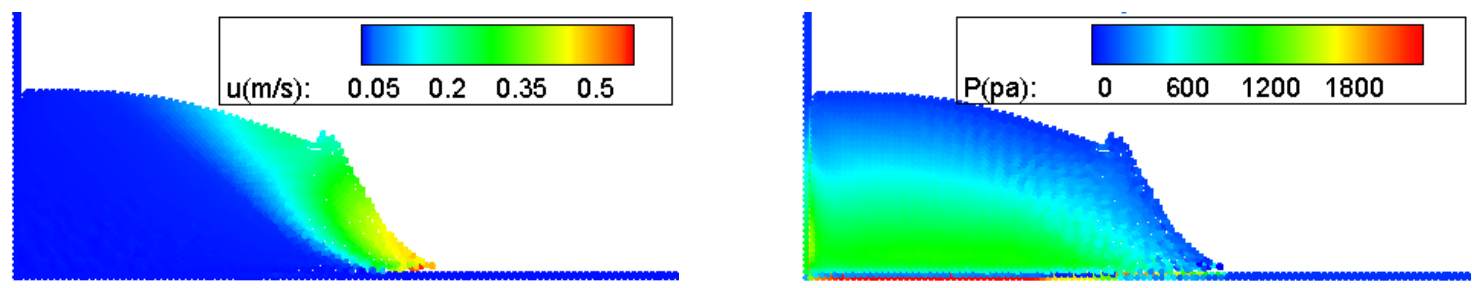

$$
t=0.12 \mathrm{~s}
$$
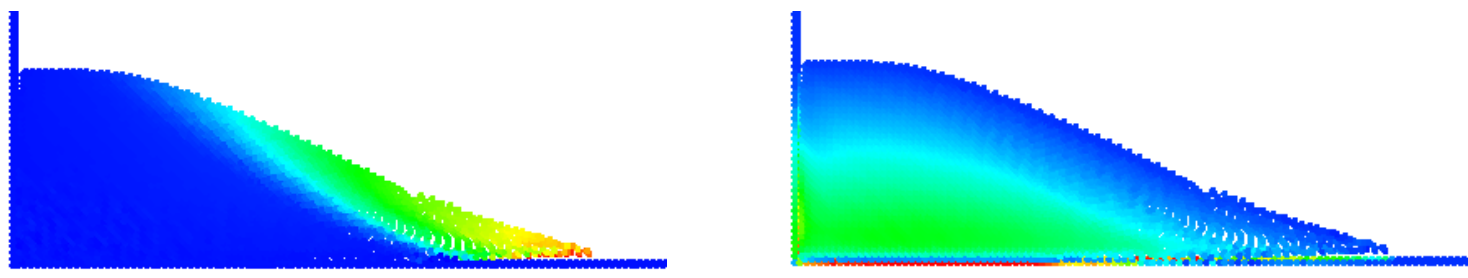

$$
t=0.2 \mathrm{~s}
$$
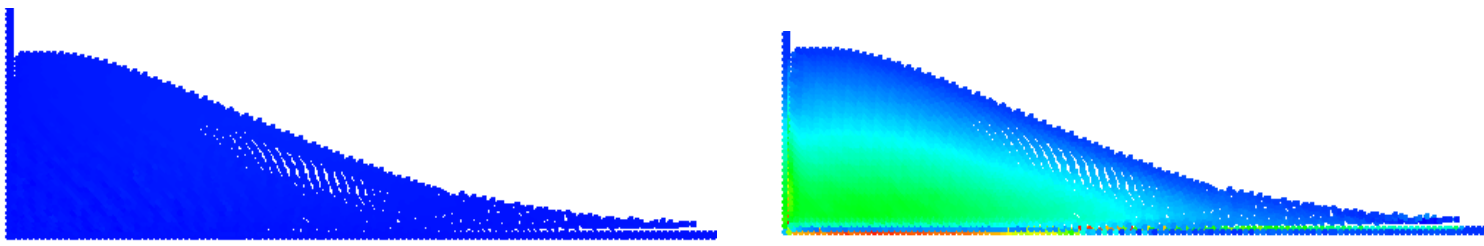

$$
t=0.4 \mathrm{~s}
$$

Fig. 6. Position of particles and pressure (Right) and velocity magnitude (Left) for different times.
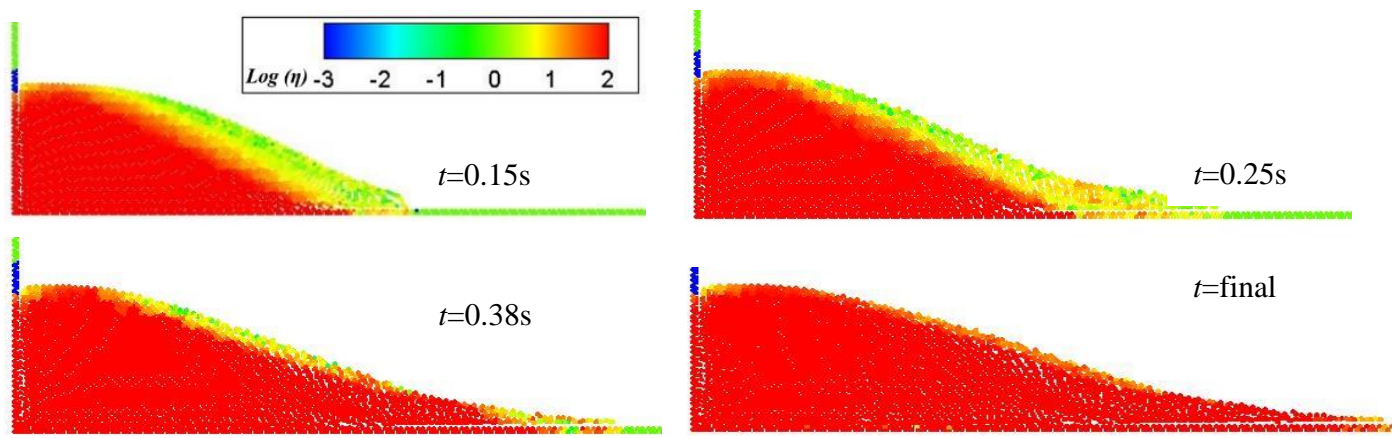

Fig. 7. Apparent viscosity changes for different times.

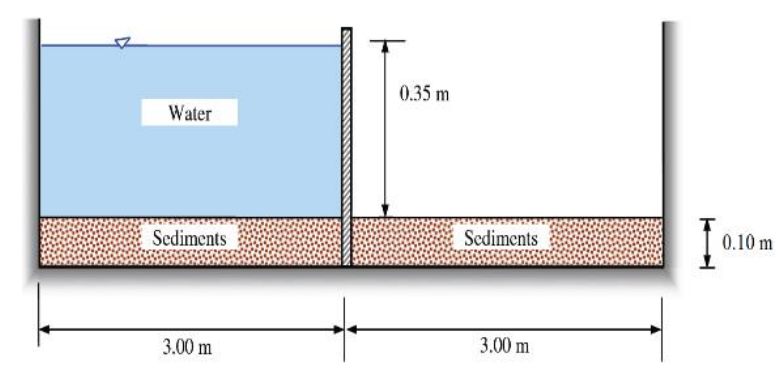

Fig. 8. A sketch view of Spinwine's setup.

$10^{-6} \mathrm{~m}^{2} / \mathrm{s}$.The saturated sediments are distributed in the bottom of flume with $6 \mathrm{~m}$ length and $0.1 \mathrm{~m}$ height. The length and depth of water in reservoir are $0.35 \mathrm{~m}$ and $3 \mathrm{~m}$, respectively. The schematic view of experimental setup is shown in Fig. 8. For the numerical simulation, the effective pressure is determined by two approaches; the method proposed by Fourtakas and Rogers (2016) and the method developed in this research, described in section 6 . The distance between the particles is $0.01 \mathrm{~m}$ in the 

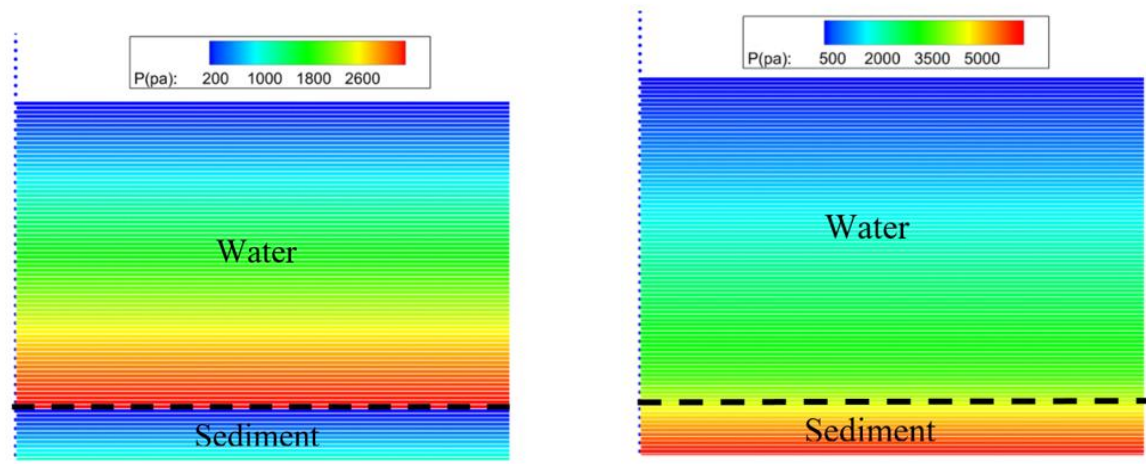

Fig. 9. Influence of pressure modification on the solid phase for SPH formulations without back pressure (left) and modified method in this research (right).
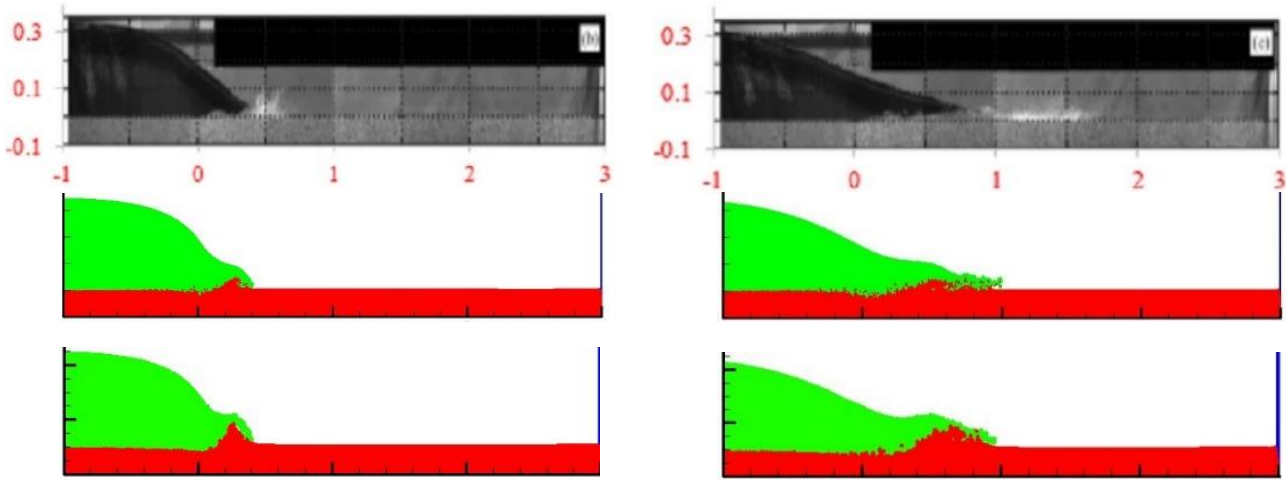

$t=0.25 \mathrm{~s}$

\section{$t=0.5 \mathrm{~s}$}
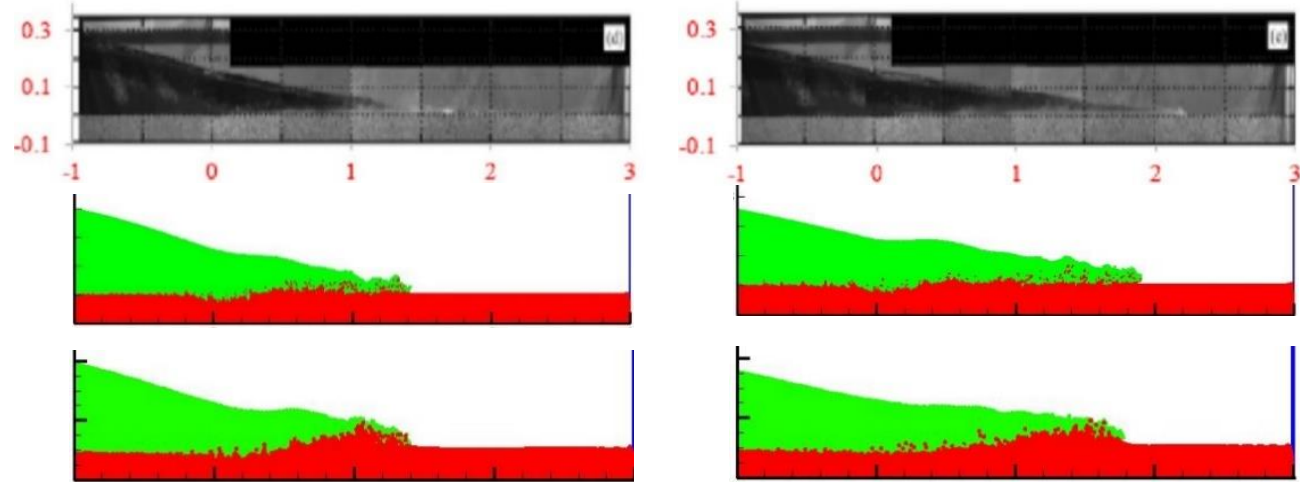

$t=0.75 \mathrm{~s}$

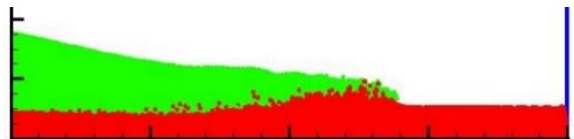

$t=1 \mathrm{~s}$

Fig. 10. Comparison the position of particles between the experimental, developed model in this research and the model proposed by Fourtakas and Rogers (2016) (up to down, respectively).

numerical model, which leads to 33708 particles. In order to simulate 1.1 seconds of the phenomenon in the physical model, the numerical simulation times were 3130 and 2868 seconds for the method developed in this research and the Fourtakas and Rogers (2016) method, respectively.

Figure 9 shows the model modified in this research (right) and the SPH formulations without back pressure (left) for determining the pressure in solid phase for the first time step in the upstream reservoir. As presented in the figure, in the unmodified model (up), there is a discontinuity in the boundary of two phases, and hence, this problem is compensated by the method developed for computing the pressure. By adding the supplementary pressure term, the pressure is modified in the body of two phases, which corresponds to the reality.

In Fig. 10, the water surface and bed deformation in the experimental and two numerical models prepared by two modification approaches for the pressure of solid phase are compared at different times. As presented in the figure, the wave formed in the start of motion leads to a gross erosion on the loose boundary. This influence is observed in the experimental and numerical models. The eroded materials are transported by water and deposited 
Table 1 Error values in different models.

\begin{tabular}{|c|c|c|c|c|c|}
\hline \multicolumn{2}{|r|}{ time } & $0.25 \mathrm{~s}$ & $0.5 \mathrm{~s}$ & $0.75 \mathrm{~s}$ & $1 \mathrm{~s}$ \\
\hline \multirow{4}{*}{$\begin{array}{l}R_{x} \text { for water } \\
\text { free surface }\end{array}$} & Current model (33708 particles) & 0.0338 & 0.0504 & 0.089 & 0.0788 \\
\hline & Current model (133303 particles) & 0.0337 & 0.0504 & 0.0885 & 0.0787 \\
\hline & Developed model (Fourtakas and Rogers, 2016) & 0.0829 & 0.0982 & 0.101 & 0.1273 \\
\hline & Ran et al. (2015) & 0.078 & 0.0831 & 0.094 & - \\
\hline \multirow{4}{*}{$\begin{array}{l}R_{x} \text { for sediment } \\
\text { surface }\end{array}$} & Current model (33708 particles) & 0.0342 & 0.0772 & 0.0649 & 0.1091 \\
\hline & Current model (133303 particles) & 0.0342 & 0.0771 & 0.0649 & 0.109 \\
\hline & $\begin{array}{c}\text { Developed model using } \\
\text { (Fourtakas and Rogers, 2016) }\end{array}$ & 0.1278 & 0.1346 & 0.1115 & 0.1603 \\
\hline & Ran et al. (2015) & 0.045 & 0.1014 & 0.1412 & - \\
\hline
\end{tabular}

downstream, as they compensate the height and velocity of negative wave formed by dam break. In this figure, the position of particles, dam break wave and its influences on the loose boundary are presented at different times. We conclude that both methods for modification of the pressure provide acceptable results, but the method developed in this research is more accurate than the method proposed by Fourtakas and Rogers (2016).

Figure 11 shows the velocity values along the flow where, initially, the sediment transport is as high as the velocity. As time passes and the water velocity is decreased, the erosion is decreased and the water movement does not cause significant changes in the sediment surface.

The superiority of the developed method for modifying the pressure in this research is shown in Fig. 12. This figure presents a comparison between the water surface and sediment surface in the experimental model and three numerical models. The method developed in the present study gives better results in comparison with the Fourtakas and Rogers (2016) method and Ran et al. (2015) model. Moreover, it is shown that the present relation for the effective pressure show better performance than the effective pressure suggested by Fourtakas and Rogers (2016). This may be due to the larger and unphysical effective pressure in the latter which causes a larger shear stresses and larger bed erosion.

An error analysis is used for investigating the performance of models. $R_{x}$ error scale is determined by Eq. (35): (Shakibaeinia and Jin, 2011):

$R_{x}=\frac{\sum_{i=1}^{N}(\Delta H)_{i}^{2}}{\sum_{i=1}^{N}(H)_{i}^{2}}$

In this relation, $\Delta H$ is the difference between the experimental free surface and the free surface of models and $H$ is the height of particle mass in the experimental model. The error values are shown in Table 1 for different time steps. As shown in the table, the error values for the present model are insignificant compared to those suggested in the Fourtakas and Rogers (2016) model. For the convergence test, the particle spacing of $0.005 \mathrm{~m}$ with 133303 particles is applied and the results are shown in Table 1.
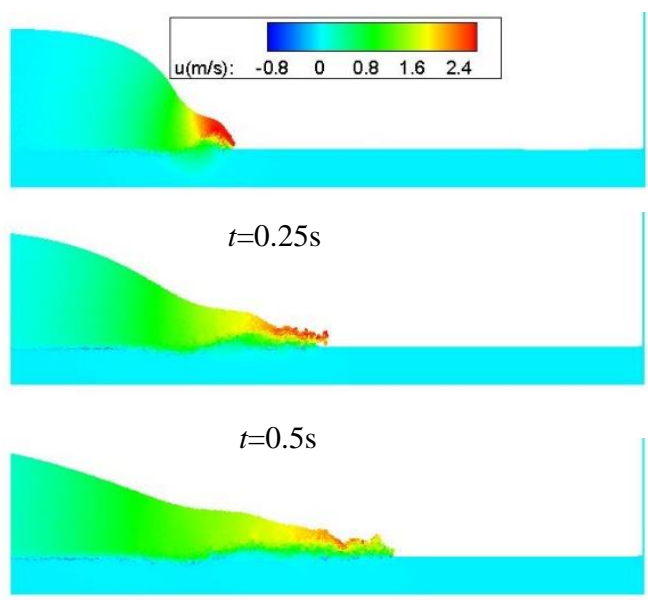

$t=0.75 \mathrm{~s}$

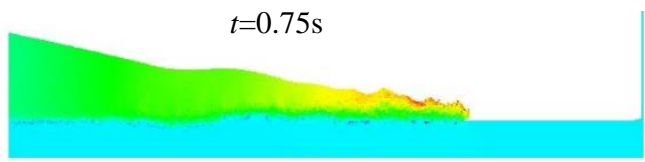

$t=1 \mathrm{~s}$

Fig. 11. Velocity magnitude $u(\mathrm{~m} / \mathrm{s})$ for different times.

\subsection{Submerged Column Collapse}

Here, the two-phase model of sediment transport is modeled in water where the numerical results are compared with the experimental data of Rondon et al. (2011). The schematic of the problem is shown in Fig. 13 where the sediment phase starts after the sudden removal of the gate. Here, $h_{o}=4.8 \mathrm{~cm}, l_{o}=$ $6 \mathrm{~cm}, h_{f}=10 \mathrm{~cm}$ and $l_{f}=24 \mathrm{~cm}$ where the dry density, bulk density and internal friction angle are $2500 \mathrm{~kg} / \mathrm{m}^{3}, 1825 \mathrm{~kg} / \mathrm{m}^{3}$ and $24.6^{\circ}$, respectively. The values in the rheological model are $I_{o}=0.279, \mu_{s}=$ $\tan 24.6^{\circ}$ and $\mu_{2}=\tan 37.1^{\circ}$ (Rondon et al. 2011; $\mathrm{Xu}$ and Jin, 2016).

Also, a mixture of water and Ucon oil $75 \mathrm{H} 90000$ from Dow was used in the experimental model with density of $1000 \mathrm{~kg} / \mathrm{m}^{3}$ and viscosity of $0.012 \mathrm{~Pa}$.s which was also used in the numerical model (Rondon et al. 2011).

The initial particle spacing is $0.001 \mathrm{~m}$, which leads to the total particle of 48283 . 
M. Kheirkhahan et al. / JAFM, Vol. 13, No. 4, pp. 1193-1206, 2020.
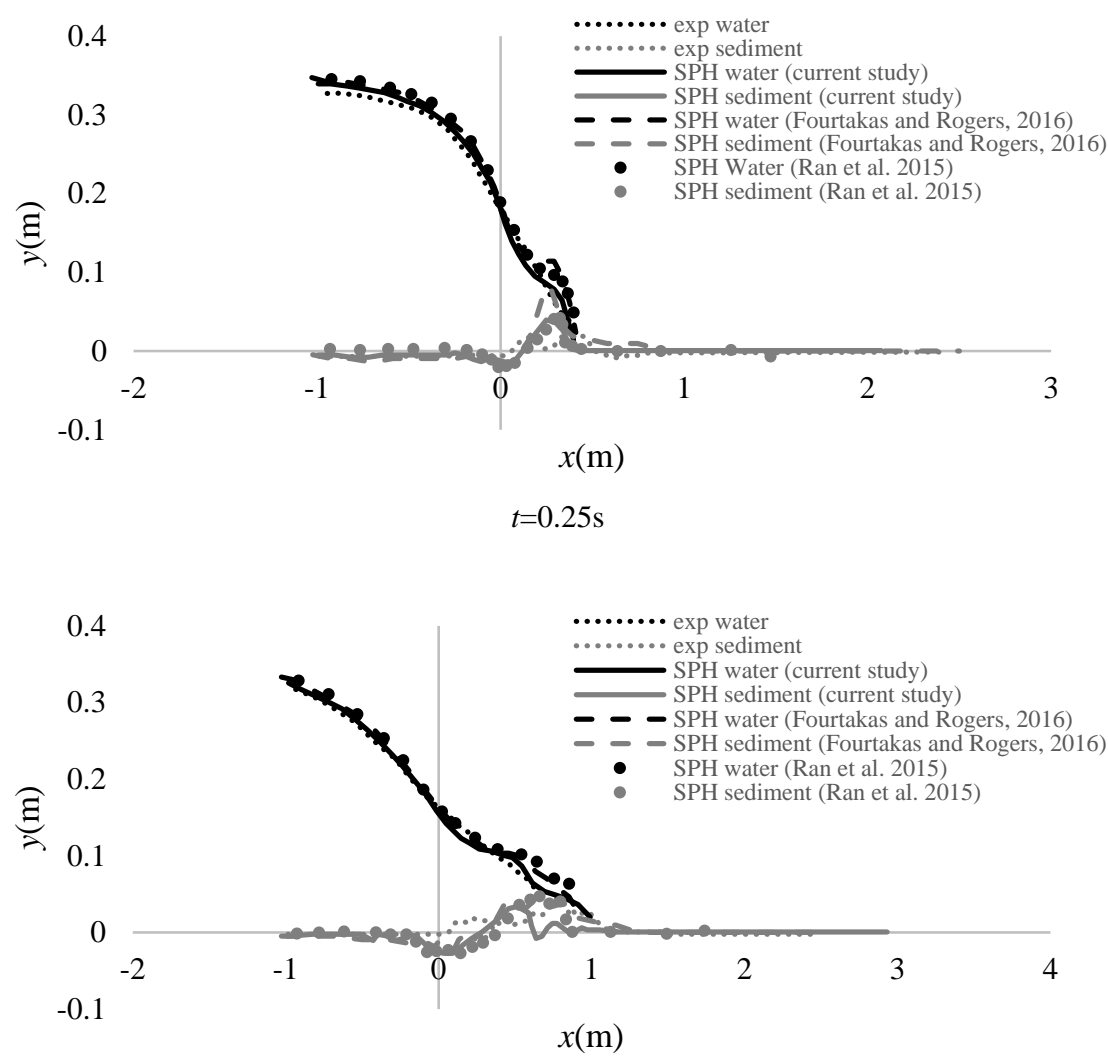

$t=0.5 \mathrm{~s}$
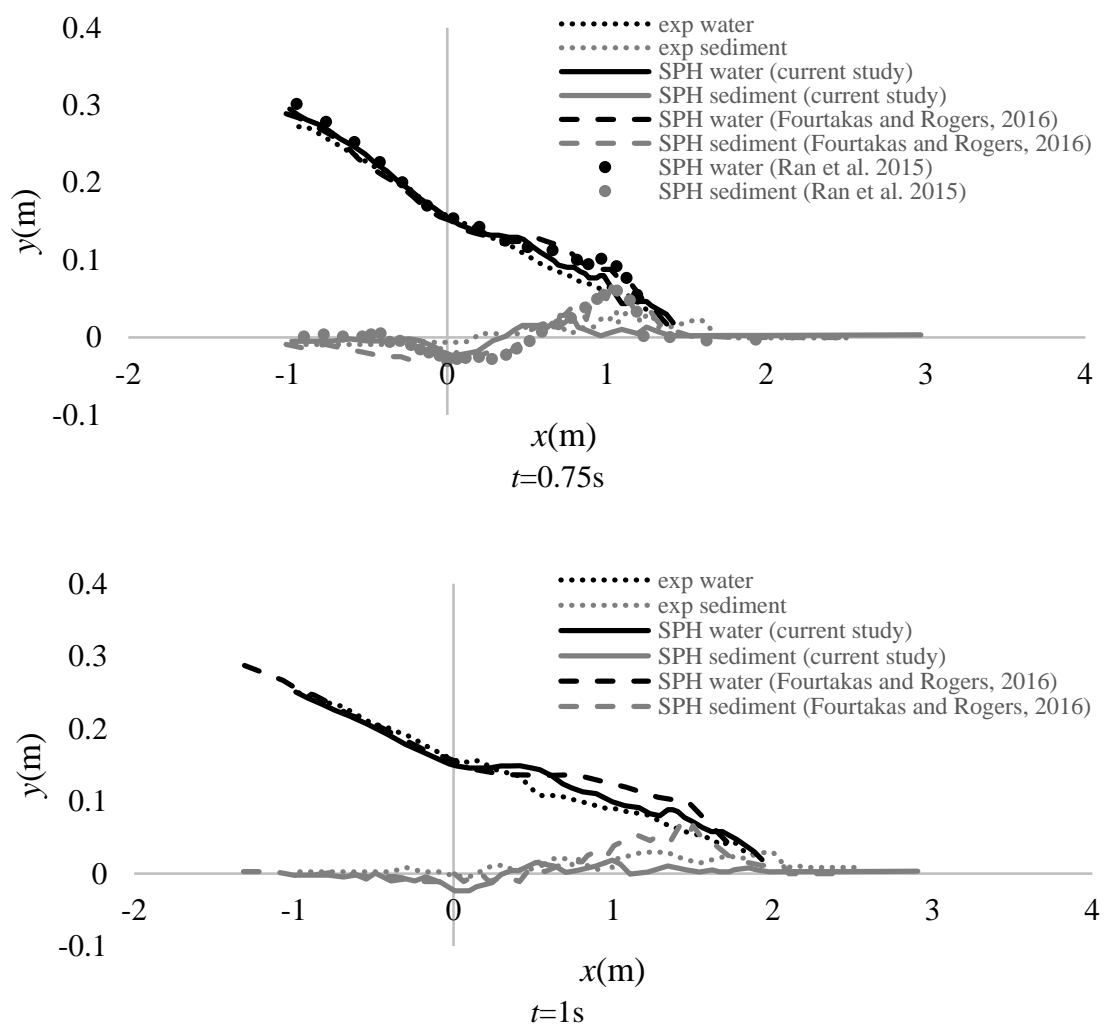

Fig. 12. Comparison water surface and sediment surface in experimental and numerical models for different times. 


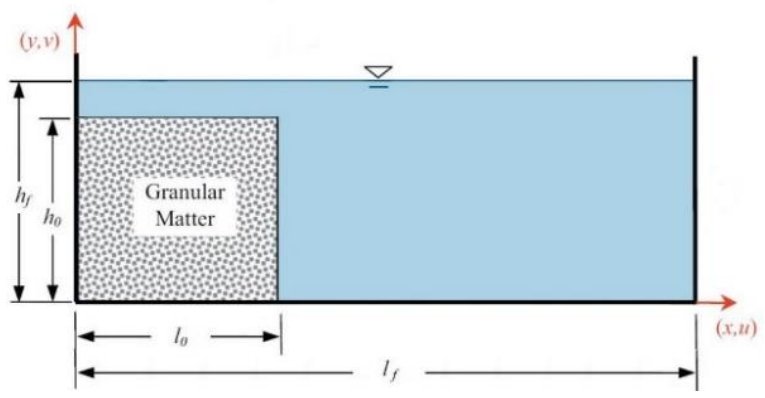

Fig. 13. A sketch view of reservoir and initial positions of particles.
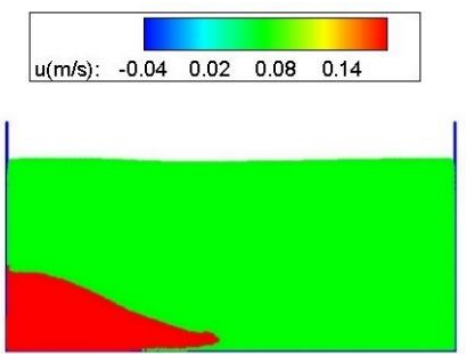

$t=40 \mathrm{~s}$
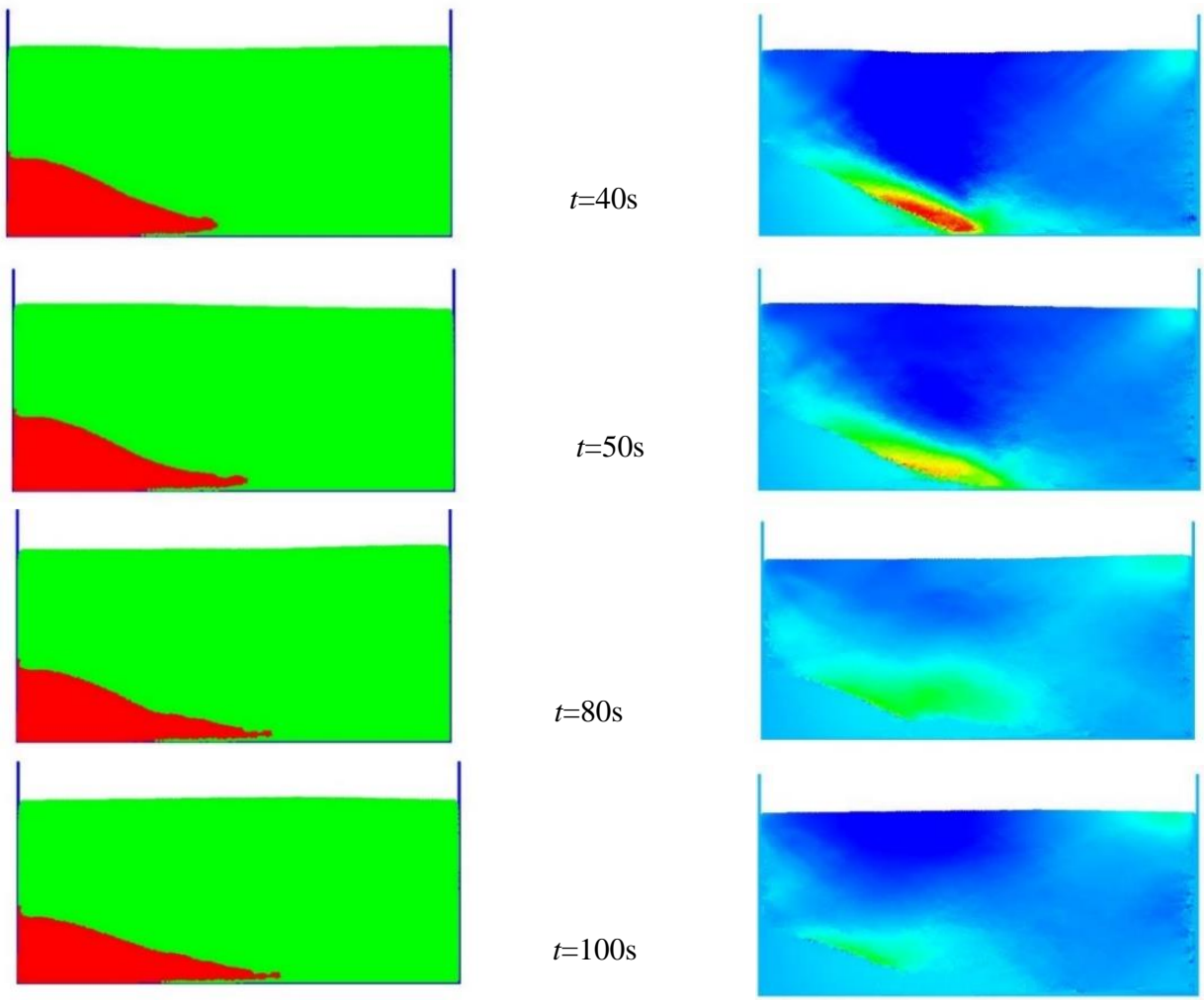

Fig. 14. Particle positions (left) and velocity magnitude $u(\mathrm{~m} / \mathrm{s})$ (right) for different times.

Figure 14 shows the particle position and the velocity values along the fluid flow $(\mathrm{u})$ at different times. As seen, initially, the particles begin to move and interacts with the soluble particles due to the weight of the particles and as a result of the pressure. Then, as time passes, the motion of particles is decreased and eventually, the sediment particles stop moving.

Figure 15 shows the surface of the sediments in the present study and the experimental model together with the numerical models of Savage et al. (2014) and Wang et al. (2017) at different times. As seen, the present study gives better results for the free surface model in comparison with the other numerical results.

\section{CONCLUSION}

This study aims to model the motion of non-cohesive particles in the absence and presence of water by SPH method. A non-Newtonian viscoplastic rheological model is implemented in SPHysics code. Some modifications are made to model the movement of the granular embankment via its own weight. The results showed that a good agreement is obtained by comparing the results with the experimental benchmark. Using the Owen equation and mean harmonic viscosity, the dam break movement phenomenon can be modeled in the same manner as the reality. The movement of granular materials in the presence of water is investigated by a two-phase model implemented in SPHysics2D 

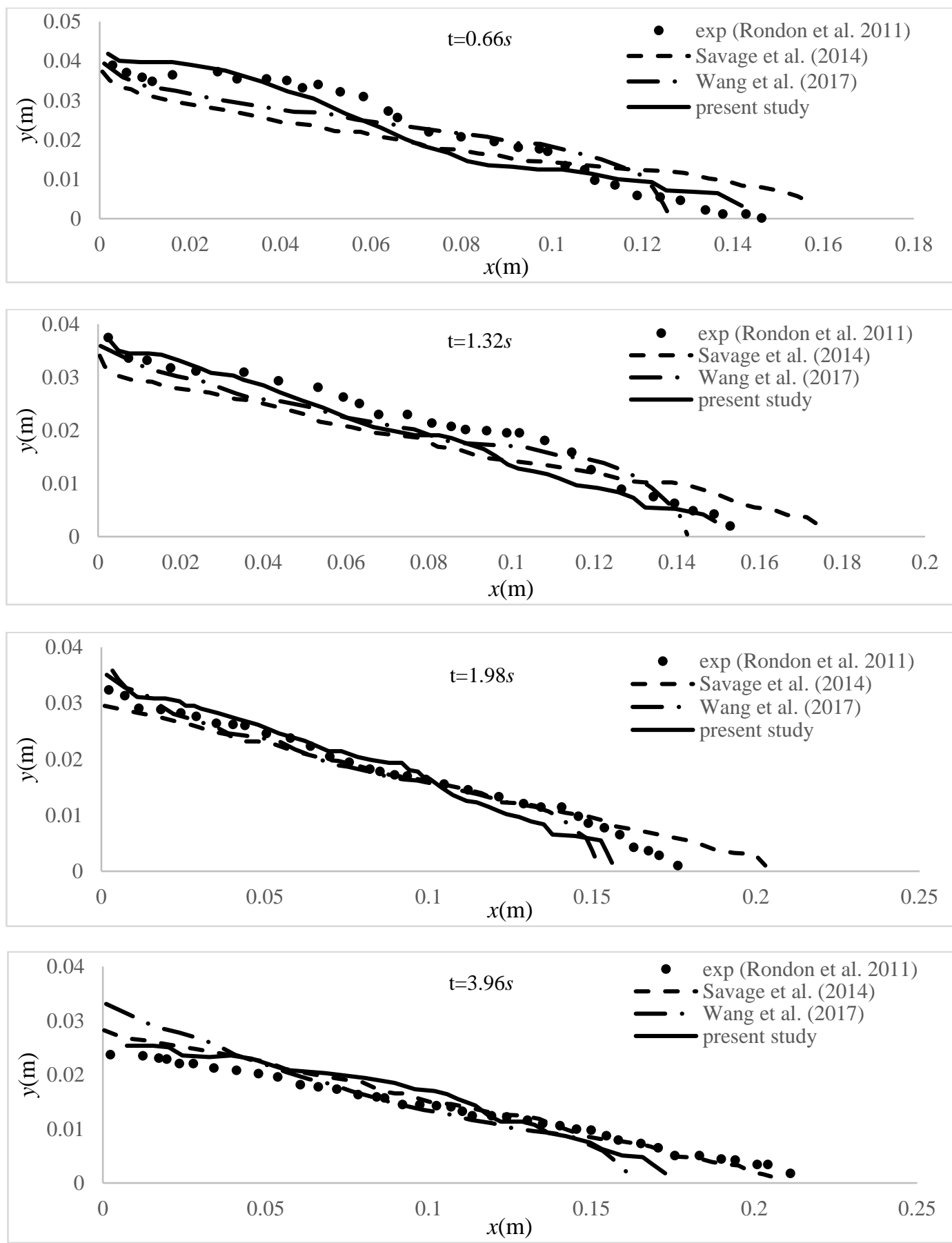

Fig. 15. Comparison sediment surface in experimental and numerical models for different times.

code. One of the interesting subjects in the behavior of saturated granular material is supporting the stresses imposed on a saturated body consisted of different phases. The modification of effective pressure on the granular material is made by two approaches. One approach was proposed by Fourtakas and Rogers (2016). The other approach was developed in this research which is consistent with the soil mechanics rules. The dam break phenomenon on an erodible bed is investigated by two approaches for determining the effective pressure in solid phase and compared with the experimental results reported by Spinwine (2005). In all times, the developed approach gives more adequate results for the experiments. Finally, the experimental model of submerged sediment mass collapse was modeled in the aquatic environment and the results of present study was compared to those of the experimental model and numerical studies of other researchers. The results showed that the developed model can be reliably used for simulating the sediment-containing models and analyzing the soil and hydraulic engineering. 


\section{REFERENCES}

Chauchat, J. and M. Médale (2014). A threedimensional numerical model for dense granular flows based on the $\mu(\mathrm{I})$ rheology. Journal of Computational Physics 256, 696712 .

Colagrossi, A. and M. Landrini (2003). Numerical simulation of interfacial flows by smoothed particle hydrodynamics. Journal of computational Physics 191(2), 448-475.

Da Cruz, F., S. Emam, M. Prochnow, J. N. Roux and F. Chevoir (2005). Rheophysics of dense granular materials: Discrete simulation of plane shear flows. Physical Review E 72(2), 021309.

Fourtakas, G. and B. D. Rogers (2016). Modelling multi-phase liquid-sediment scour and resuspension induced by rapid flows using Smoothed Particle Hydrodynamics (SPH) accelerated with a Graphics Processing Unit (GPU). Advances in Water Resources 92, 186199.

Fu, L. and Y. C. Jin (2016). Improved multiphase Lagrangian method for simulating sediment transport in dam-break flows. Journal of Hydraulic Engineering 142(6), 04016005.

Gingold, R. A. and J. J. Monaghan (1977). Smoothed particle hydrodynamics: theory and application to non-spherical stars. Monthly Notices of the Royal Astronomical Society 181(3), 375-389.

Gómez-Gesteira, M., A. J. Crespo, B. D. Rogers, R. A. Dalrymple, J. M. Dominguez and A. Barreiro (2012). SPHysics-development of a free-surface fluid solver-Part 2: Efficiency and test cases. Computers and Geosciences 48, 300307.

Grenier, N., M. Antuono, A. Colagrossi, D. Le Touzé and B. Alessandrini (2009). An Hamiltonian interface SPH formulation for multi-fluid and free surface flows. Journal of Computational Physics 228(22), 8380-8393.

Hampton, M. A., H. J. Lee and J. Locat (1996). Submarine landslides. Reviews of geophysics 34(1), 33-59.

Hosseini, K., P. Omidvar, M. Kheirkhahan and S. Farzin (2019). Smoothed particle hydrodynamics for the interaction of Newtonian and non-Newtonian fluids using the $\mu$ (I) model. Powder Technology 351, 325-337.

Hosseinkhani, M. R. P. and Omidvar (2018). Smoothed Particle Hydrodynamics for the rising pattern of oil droplets. Journal of Fluids Engineering 140(8), 081105.

Iverson, R. M. (1997). The physics of debris flows. Reviews of geophysics 35(3), 245-296.

Jop, P., Y. Forterre and O. Pouliquen (2005). Crucial role of sidewalls in granular surface flows: consequences for the rheology. Journal of Fluid Mechanics 541, 167-192.
Khanpour, M., A. R. Zarrati, M. Kolahdoozan, A. Shakibaeinia and S. M. Amirshahi (2016). Mesh-free SPH modeling of sediment scouring and flushing. Computers and Fluids 129, 67-78.

Kheirkhahan, M. and K. Hosseini (2018). Comparison of the $\mu$ (I) and HBP models for simulating granular media. International Journal of Modern Physics C 29(07), 1850050.

Lajeunesse, E., J. B. Monnier and G. M. Homsy (2005). Granular slumping on a horizontal surface. Physics of Fluids 17(10), 103302.

Legros, F. (2002). The mobility of long-runout landslides. Engineering geology 63(3-4), 301331.

Lo, E. Y. and S. Shao (2002). Simulation of nearshore solitary wave mechanics by an incompressible SPH method. Applied Ocean Research 24(5), 275-286.

Lucy, L. B. (1977). A numerical approach to the testing of the fission hypothesis. The astronomical Journal 82, 1013-1024.

Meruane, C., A. Tamburrino O. and Roche (2010). On the role of the ambient fluid on gravitational granular flow dynamics. Journal of Fluid Mechanics 648, 381-404.

Meruane, C., A. Tamburrino and O. Roche (2012). Dynamics of dense granular flows of small-andlarge-grain mixtures in an ambient fluid. Physical Review E 86(2), 026311.

MiDi, G. D. R. (2004). On dense granular flows. The European Physical Journal E 14(4), 341-365.

Monaghan, J. J. (1994). Simulating free surface flows with SPH. Journal of computational physics 110(2), 399-406.

Monaghan, J. J. (2005). Smoothed particle hydrodynamics. Reports on progress in physics 68(8), 1703.

Monaghan, J. J. and Kos, A. (1999). Solitary waves on a Cretan beach. Journal of waterway, port, coastal, and ocean engineering 125(3), 145155.

Morris, J. P., P. J. Fox and Y. Zhu (1997). Modeling low Reynolds number incompressible flows using SPH. Journal of computational physics 136(1), 214-226.

Nikeghbali, P. and P. Omidvar (2018). Application of the SPH Method to Breaking and Undular Tidal Bores on a Movable Bed. Journal of Waterway, Port, Coastal, and Ocean Engineering 144(2), 04017040.

Omidvar, P. (2010). Wave loading on bodies in the free surface using Smoothed Particle Hydrodynamics (SPH) (Doctoral dissertation, The University of Manchester (United Kingdom)).

Omidvar, P. and P. Nikeghbali (2017). Simulation of violent water flows over a movable bed using smoothed particle hydrodynamics. Journal of 
M. Kheirkhahan et al. / JAFM, Vol. 13, No. 4, pp. 1193-1206, 2020.

Marine Science and Technology 22(2), 270287.

Omidvar, P., O. Farghadani and P. Nikeghbali (2017). SPH for impact force and ricochet behavior of water-entry bodies. International Journal of Modern Physics C 28(10), 1750119.

Omidvar, P., M. Kheirkhahan and Kh. Hosseini (2018). Development of Smoothed Particle Hydrodynamics for simulating two-phase water-sediment flow using $\mu(\mathrm{I})$ rheological model. Modares Mechanical Engineering 18(8), 173-182.

Omidvar, P., H. Norouzi and A. Zarghami (2015). Smoothed particle hydrodynamics for water wave propagation in a channel. International Journal of Modern Physics C 26(08), 1550085.

Papanastasiou, T. C. (1987). Flows of materials with yield. Journal of Rheology 31(5), 385-404.

Ran, Q., J. Tong, S. Shao, X. Fu and Y. Xu (2015). Incompressible SPH scour model for movable bed dam break flows. Advances in Water Resources 82, 39-50.

Rogers, B. D., R.A. Dalrymple and P. K. Stansby (2009). SPH modeling of floating bodies in the surf zone. In Coastal Engineering 2008: (In 5 Volumes) (pp. 204-215).

Rondon, L., O. Pouliquen and P. Aussillous (2011). Granular collapse in a fluid: role of the initial volume fraction. Physics of Fluids 23(7), 073301 .

Savage, S. B., M. H. Babaei T. and Dabros (2014). Modeling gravitational collapse of rectangular granular piles in air and water. Mechanics Research Communications 56, 1-10.

Shadloo, M. S., G. Oger D. and Le Touzé (2016). Smoothed particle hydrodynamics method for fluid flows, towards industrial applications: Motivations, current state, and challenges. Computers and Fluids 136, 11-34.

Shakibaeinia, A. and Y. C. Jin (2011). A mesh-free particle model for simulation of mobile-bed dam break. Advances in Water Resources 34(6), 794-807.

Shao, S. and E. Y. Lo (2003). Incompressible SPH method for simulating Newtonian and nonNewtonian flows with a free surface. Advances in Water Resources 26(7), 787-800.

Spinewine, B. (2005). Two-layer flow behaviour and the effects of granular dilatancy in dam-break induced sheet-flow. PhD diss., Faculté des sciences appliquées, Université catholique de Louvain.

Szewc, K. (2017). Smoothed particle hydrodynamics modeling of granular column collapse. Granular Matter 19(1), 3.

Wang, C., Y. Wang, C. Peng and X. Meng (2017). Two-fluid smoothed particle hydrodynamics simulation of submerged granular column collapse. Mechanics Research Communications 79, 15-23.

Xu, T. and Y. C. Jin (2016). Modeling free-surface flows of granular column collapses using a mesh-free method. Powder Technology 291, 20-34. 